\title{
Lokaltherapie der Psoriasis
}

\section{R. Gillitzer}

\section{Topical Therapy of Psoriasis}

Zusammenfassung

Primäres Ziel der Psoriasistherapie ist es, über eine Reduktion der befallenen Körperareale die komplette Abheilung mit einem möglichst langen rezidivfreien Intervall zu erreichen. In den meisten Fällen kann dies durch eine topische Therapie, manchmal in Kombination mit einer UV-Lichttherapie, erreicht werden. Nur bei schweren oder therapierefraktären Krankheitsverläufen ist eine systemische Therapie notwendig.

Das umfangreiche Armamentarium an Lokaltherapeutika umfasst Keratolytika, Dithranol (Anthralin, Cignolin), Vitamin$\mathrm{D}_{3}$-Analoga, topische Steroide, Tazarotene, Teerpräparate und einige weitere Substanzen, die sich aufgrund ihrer unterschiedlichen Wirkweise sinnvoll kombinieren lassen. Dadurch wird eine deutliche Wirkungsverbesserung bei gleichzeitiger Reduktion der Nebenwirkungen erreicht. Bei der Wahl der Substanzen sollte darauf geachtet werden, dass Substanzen, die vornehmlich die Hyperproliferation und Akanthose beeinflussen (Vitamin$\mathrm{D}_{3}$-Analoga, Tazaroten), mit Substanzen, die die Entzündung hemmen (Steroide), und/oder mit UV-Licht kombiniert werden. Trotz eines detaillierteren Wissens über wichtige Pathogeneseschritte der Psoriasis und der Wirkungsweise der meisten Lokaltherapeutika fehlen bis heute aber klare evidenzbasierte Therapieleitlinien, die die Entscheidung für eine maßgeschneiderte individuelle Therapie erleichtern. Deshalb sind umfangreiche vergleichende Therapiestudien dringend notwendig, damit die besten Therapieprinzipien herausgearbeitet werden können. Nach fast neunzigjähriger Anwendung stellt Cignolin bis heute eines der effizientesten Therapeutika dar, an dem sich alle anderen antipsoriatisch wirkenden Medikamente und Medikamentenkombinationen unter sozioökonomischen Aspekten, hinsichtlich Lebensqualitätsverbesserung, Effektivität und einem möglichst langem rezidivfreien Intervall messen lassen müssen.
Abstract

The goal of psoriasis treatment is to achieve control of the disease process and to decrease the area of body surface involvement. Finally, for the majority of cases complete clearance and the maintenance of long-term remission is a realistic expectation of psoriasis treatment. Topical treatment, sometimes in combination with UV phototherapy, is sufficient in most cases of mild and moderate psoriasis and only severe cases require systemic treatment. Nowadays our armamentarium of topical drugs includes keratolytics, anthralin (dithranol, cignolin), calcipotriene, topical corticosteroids, tazarotene, topical tar and some other less effective substances. The variety of topical therapy regimes and their different modes of action allow to combine different therapy modalities thus increasing efficacy and reducing side effects as compared to monotherapy. The combination should be selected in a way that a drug with predominant anti-inflammatory activities such as corticosteroids and/or UV-light is combined with a drug with anti-proliferative activities (calcipotriene, tazarotene). Despite our detailed knowledge on psoriasis pathogenesis and the role of different drugs good evidence-based guidelines on best current practice are still missing. Therefore, well established comparative therapy studies are urgently needed to provide the most effective care for the patient. The goal has to be the achievement and maintenance of long-term remission. In this respect, therapy with anthralin is since almost ninety years still the golden standard of psoriasis therapy. Therefore, all other topical treatments and therapy combinations have to be compared with anthralin under the aspects of socio-economy, effectiveness, long-term remission and life quality. 
Einleitung

Die Psoriasis wird heute als eine (auto)immungenetische Erkrankung mit epidermaler Hyperproliferation und Entzündungsreaktion unter wesentlicher Beteiligung von T-Helfer-1-Zellen verstanden. Uns sind die Wege der Entstehung des Psoriasisherdes in vielen Schritten durch intensive Forschungsanstrengungen mittlerweile gut bekannt, wir kennen aber nicht die zugrunde liegenden Ursachen. Somit bleibt dem Patienten mit Psoriasis bis heute eine kausale Therapie, die ihn vor immer wieder auftretenden erythrosquamösen Plaques mit all ihren subjektiven und psychosozialen Problemen befreit, vorenthalten. Insofern ist die allenthalben propagierte Euphorie über die modernen systemischen Antipsoriatika, allen voran die Biologicals, zumindest unter rationalen Gesichtspunkten nicht ganz nachvollziehbar. Wenngleich für sehr schwere und komplizierte Psoriasisfälle Biologicals eine Bereicherung des oft aufgrund von Begleiterkrankungen eingeschränkten Therapiespektrums bedeuten, stoßen sie schon aufgrund der hohen Therapiekosten und notwendigen langfristigen Anwendung an sozioökonomische Grenzen.

Da die Psoriasis vorwiegend eine Hauterkrankung ist, bleibt deshalb die Lokaltherapie der wesentliche und unverzichtbare Bestandteil der Psoriasisbehandlung, mit der bei sachgemäßer und situationsadaptierter Anwendung eine meist komplette und nebenwirkungsarme Abheilung leichter bis mittelschwerer Formen erzielt werden kann. Selbst bei schweren Psoriasisformen werden unter Einsatz systemischer Therapien diese oft mit einer topischen Therapiemaßnahme kombiniert, um die Therapieeffizienz zu verbessern und Nebenwirkungen zu minimieren. Insofern ist die Lokaltherapie essentiell für die Behandlung aller Schweregrade der Psoriasis, was ihren grundsätzlichen Stellenwert unterstreicht.

Im Folgenden sollen die einzelnen Lokalbehandlungsprinzipien dargestellt, verglichen und bewertet werden. Dabei soll im Wesentlichen die Plaquepsoriasis Gegenstand der Therapieempfehlungen sein. Soweit entsprechende Therapiemodifikationen bei unterschiedlichen Lokalisationen oder Sonderformen der Psoriasis (intertriginöser Befall, pustulöse Formen, Psoriasis im Kindesalter) notwendig sind, wird darauf verwiesen.

\section{Abschuppende und pflegende Maßnahmen}

Die Psoriasisplaque ist durch eine ausgeprägte Akanthose und Hyperkeratose charakterisiert, die den eigentlichen Entzündungsprozess überdeckt. Bereits einfache pflegerische Maßnahmen führen zu einer deutlichen Befundbesserung (blande Keratolyse) [1]. Deshalb sollte dem Patienten vermittelt werden, dass zur erfolgreichen Lokaltherapie eine intensive Pflege der Läsionen einen wichtigen Beitrag leistet. Je nach Jahreszeit, Lokalisation und Bedürfnis des Patienten sollten Basiscremes oder Basissalben mehrmals täglich aufgetragen werden. Empfehlenswert ist die zusätzliche Pflege des Integuments mit einer W/O-Emulsion mindestens einmal täglich und das Meiden von zu häufigem Wasserkontakt und Detergentien (Duschen, Duschgels), die zu einer Austrocknung der ohnehin trockenen Plaques und verstärktem Juckreiz führen. Wenngleich der Juckreiz nicht ein Kardinalsymptom wie etwa beim atopischen Ekzem darstellt, leiden nicht wenige Psoriatiker bei unzureichender Pflege unter Pruritus. Pflegende Maßnahmen dienen auch der Prophylaxe im rezidivfreien Intervall und sollten deshalb regelmäßig und konsequent durchgeführt werden.

Da die ausgeprägte Schuppung der Psoriasis (Hyper- und Parakeratose) ein Hindernis für eine weitere differente topische Therapie oder Lichttherapie darstellt, ist die läsionale Keratolyse zu Beginn der Lokalbehandlung obligat. Keratolytische Effekte zeigt insbesondere Salizylsäure (5-10\%), die die Kittsubstanz der Hornschicht lockert und deren Ablösung bewirkt. Dadurch wird eine sinnlose Bindung von Arzneistoffen an Hornzellen vermieden und gleichzeitig die Einschleusung anderer Substanzen erleichtert (doppelte indirekte Absorptionsbahnung). Da die Psoriasisplaque meist eine sehr trockene Läsion ist, sollte die Salizylsäure in Salbengrundlagen rezeptiert werden. Bewährt und kostengünstig ist die Rezeptur in Vaseline. Zu berücksichtigen ist, dass die Wirkung der Salizylsäure vehikelabhängig ist. Aus Vaseline wird wenig Salizylsäure freigesetzt, mehr aus Ölen und Lipogelen, weshalb entsprechend höhere bzw. niedrigere Konzentrationen in die Grundlage eingemischt werden müssen. Aufgrund der irritativen Wirkung von Salizylsäure und bei meist fehlender Akanthose und Hyperkeratose in intertriginösen Arealen sollte ein Salizylsäure-haltiges Externum dort nicht eingesetzt werden und der Patient explizit darüber aufgeklärt werden. Bei großflächiger Anwendung muss beachtet werden, dass Salizylsäure insbesondere bei Kindern zur Intoxikation führen kann (neuro- und nephrotoxisch), weshalb Konzentrationen bis 3\% empfohlen werden [2]. Bei Säuglingen sollte die Anwendungsfläche unter $5 \%$, bei älteren Kindern unter 20\% der Körperoberfläche liegen [3]. Deshalb sind Salizylsäure-haltige Externa bei Säuglingen, Kleinkindern und Schwangeren bei großflächiger und höher konzentrierter Anwendung kontraindiziert (siehe auch Artikel von Benoit und Goebeler in dieser Ausgabe der Aktuellen Dermatologie). Es wird deshalb ein äußerst sparsamer und vorsichtiger Gebrauch bei Säuglingen und Kleinkindern empfohlen. Eine wenngleich geringere keratolytische Wirkung hat Harnstoff, der bei empfindlichen Patienten anstelle von Salizylsäure verwendet werden kann (bis 12\% Harnstoff). Bewährt hat sich auch die Kombination von Salizylsäure und Harnstoff in einer Salbengrundlage (z. B. $5 \%$ Salizylsäure in $10 \%$ Harnstoffsalbe, z. B. Kerasal $\left.{ }^{\circledR}\right)$. Unter dem Hinweis „Psoriasis“ sollten Salizylsäure-haltige Externa für die Psoriasistherapie auf Kassenrezept verordnet werden, da der Patient als chronisch Kranker bereits die Kosten für die notwendigen Pflegeprodukte (leider auch Harnstoffpräparate) selbst zu tragen hat.

Keratolytische Wirkung und Abschuppung kann auch durch Sole-Bäder und Meerwasser, insbesondere bei hoher Salzkonzentration (Totes Meer), erreicht werden. Kochsalzhaltige Cremes sind allerdings in ihrer abschuppenden Wirkung im Vergleich zur Salizylsäure deutlich schwächer.

Die abschuppenden Maßnahmen sollten so lange durchgeführt werden, bis die Plaques weitgehend schuppenfrei und homogen rot erscheinen und die weiteren Lokaltherapeutika und/oder therapeutisches Licht (siehe Artikel von U. Hofmann in dieser Ausgabe der Aktuellen Dermatologie) ungehindert in die tieferen Epidermis- bzw. Hautschichten eindringen können. Gewöhnlich dauert dies unter Anwendung Salizylsäure-haltiger Präparate bei 
mehrmals täglichem Auftragen 3-5 Tage. Bei der Anwendung von Salizylsäure-haltigen Salben zur Abschuppung ist unbedingt darauf zu achten, dass Calcipotriol-haltige Cremes und Salben nicht unmittelbar danach eingesetzt werden, da Salizylsäure die Wirkung von Calcipotriol hemmt [4]. Deshalb empfiehlt sich der intermittierende Einsatz von Harnstoffsalben für 2-3 Tage nach Anwendung von Salizylsäure und vor Anwendung von Vitamin$\mathrm{D}_{3}$-Analoga.

\section{Vitamin- $\mathbf{D}_{3}$-Analoga}

Die antipsoriatische Wirkung eines Vitamin- $\mathrm{D}_{3}$-Präparates wurde erstmals 1985 nachgewiesen [5]. Derzeit stehen 3 Vitamin- $\mathrm{D}_{3}$ Präparate für die Lokaltherapie zur Verfügung: Calcipotriol (Daivonex $^{\circledR}$, Psocutan ${ }^{\circledR}$ ), Calcitriol $\left(\right.$ Silkis $^{\circledR}$ ) und Tacalcitol (Curato$\operatorname{derm}^{\circledR}$ ). Sie wirken über einen Kernrezeptor, was über verschiedene Zwischenschritte zu einer Expression von Genen führt, die die psoriasistypische Hyperproliferation und Keratinisation hemmen. Zusätzlich wurden entzündungshemmende Effekte nachgewiesen. Von klinischer Seite steht aber die proliferationshemmende Wirkung eindeutig im Vordergrund. Erfahrungsgemäß kommt es nach Anwendung sehr bald zu einer Abnahme der Schuppung und einer deutlichen Abflachung der Plaques, während die Rötung und ihr mikroskopisches Korrelat, die Entzündung, persistiert. Deshalb wird die Anwendung von Vitamin$\mathrm{D}_{3}$-Analoga mit anderen Therapieverfahren, die antientzündlich wirken, erfolgreich kombiniert. Allen voran hat sich die Kombination mit topischen Steroiden (siehe unten) und Lichttherapie (siehe Artikel von U. Hofmann) als erfolgreich und der jeweiligen Monotherapie als deutlich überlegen erwiesen. Das gelegentlich geringe Ansprechen von Vitamin- $\mathrm{D}_{3}$-Analoga bei einzelnen Psoriatikern wird mit einem möglichen Vitamin- $\mathrm{D}_{3}$-Rezeptorpolymorphismus erklärt, der dazu führt, dass bei manchen Patienten die Wirkung deutlich geringer ausfällt [6]. Bei Nichtansprechen der Therapie sollte deshalb auf andere Antipsoriatika gewechselt werden.

Von den drei derzeit verfügbaren Vitamin- $\mathrm{D}_{3}$-Analoga wird Calcipotriol am häufigsten verwendet und in einigen klinischen Studien wurde eine geringfügige Überlegenheit von Calcipotriol nachgewiesen [7]. Zur Behandlung steht Calcipotriol (Daivonex ${ }^{\circledR}$, Psorcutan ${ }^{\circledR}$ ) in verschiedenen galenischen Aufbereitungen (Lösung, Creme, Salbe) zur Verfügung, so dass auch Problemzonen (behaarter Kopf, Intertrigines) adäquat behandelt werden können.

Um eine negative Beeinflussung des Kalziumstoffwechsels zu vermeiden, sollte die jeweils empfohlene Tageshöchstdosis der topischen Vitamin- $\mathrm{D}_{3}$-Analoga nicht überschritten werden. Diese liegt z. B. bei Tacalcitol bei $30 \mathrm{~g} /$ Tag und bei Calcipotriol bei 15 g/Tag Fertigpräparat [8,9] und sollte als Richtmaß 30\% der Gesamtkörperoberfläche nicht überschreiten. Dies entspräche, um die pharmakologischen Dimensionen zu verdeutlichen, bei Calcipotriolpräparaten einer applizierten Menge reiner Substanz von ca. $5 \mathrm{mg} /$ Woche. Trotz eines bei sachgemäßer Anwendung geringen Risikos empfiehlt sich bei längerer großflächiger Anwendung eine gelegentliche Bestimmung des Serumkalziumspiegels. Zusammengefasst steht uns heute mit den Vitamin$D_{3}$-Analoga ein sicheres und effizientes Lokaltherapeutikum zur
Verfügung, dessen Stärken insbesondere auch in der Kombination mit Steroiden und Lichttherapie zum Tragen kommen.

\section{Topische Kortikoide}

Die topische Therapie der Psoriasis mit Steroiden ist von ihrer Effektivität und Schnelligkeit des Wirkungseintritts sowohl für den Patienten wie auch für den Dermatologen verlockend. Deshalb werden wohl unter den Antipsoriatika topische Steroidpräparate weltweit am häufigsten verwendet. Ob dieser nicht selten unkritische Einsatz gerechtfertigt ist, darf in Zweifel gezogen werden. Aufgrund der Chronizität der Psoriasis mit oft über Jahrzehnte notwendiger Lokaltherapie ist die glukokortikoidassoziierte Hautatrophie ein großes Problem (Abb.1). Bei großflächiger Anwendung sollten auch die systemischen Nebenwirkungen der Steroide berücksichtigt werden (Hypothalamus-HypophysenNNR-Achse). Was den Sinn der großflächigen und langfristigen Lokaltherapie mit Steroiden weiter einschränkt, sind die schnellen Rezidive nach Absetzen derselben (Relapse), wobei die Psoriasis nicht selten stärker wieder aufflammen kann als der ursprüngliche Befund (Rebound-Effekt). Für eine klassische Psoriasis ist deshalb die isolierte Therapie mit topischen Steroiden in Anbetracht von Alternativen und Kombinationen mit anderen Antipsoriatika nur in Ausnahmefällen (schnelle Therapie aus kosmetischen Gründen) empfehlenswert und sicherlich keine „First Line Therapie“. Lediglich intertriginöse Areale, insbesondere im Bereich der Rima ani, erlauben oft keinen Einsatz irritierender Substanzen (Salizylsäure, Dithranol) oder Licht, weshalb dort zwangsläufig aus Mangel an besseren Alternativen Steroide sinnvoll sein können. Wenngleich neuere Steroide (z. B. Mometasonfuroat, Prednicarbat) ein geringeres atrophogenes Potenzial aufweisen, sind in den Intertrigines durch die teils okklusive Situation auch die Nebenwirkungen (Atrophie) stärker (Abb.1). Dermatologen sollten dieses von Hausärzten oft überbeanspruchte Therapeutikum deshalb sehr differenziert einsetzen. Weitere Domänen lokaler Steroide sind ferner der behaarte Kopf (geringes Hautatrophierisiko) und z.B. palmoplantare Psoriasisformen, wo aufgrund oft starker Akanthose und Hyperkeratose eine quasi „dosierte Atrophie“ wünschenswert sein kann.

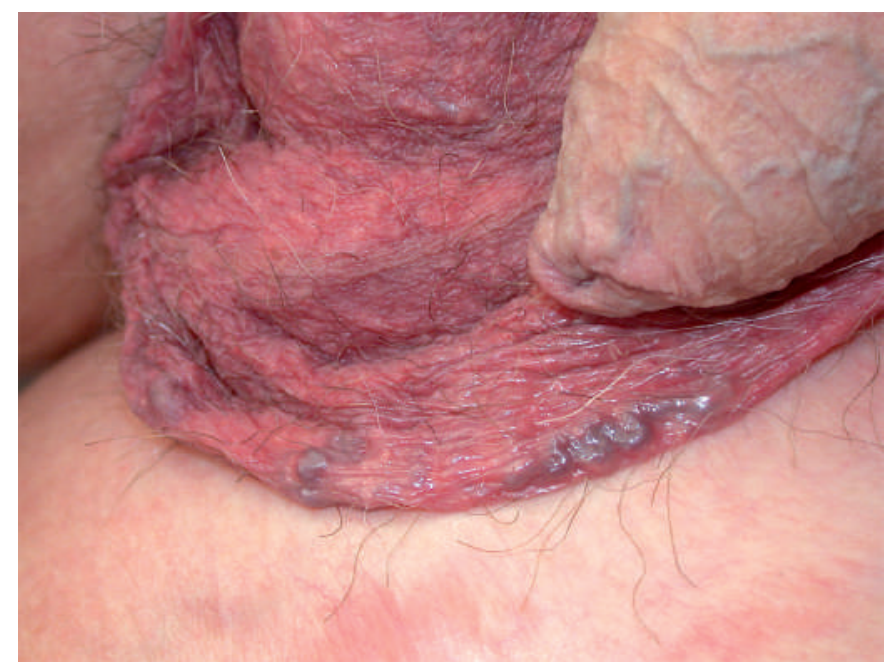

Abb. 1 Schwere Atrophie der Skrotalhaut nach jahrelanger Anwendung von lokalen Steroiden bei intertriginöser Psoriasis. 
Lokale Steroide sind in sinnvoller Kombination mit anderen Antipsoriatika eine wichtige Bereicherung des Behandlungsspektrums. Insbesondere die Kombination mit Vitamin- $\mathrm{D}_{3}$-Analoga ist pharmakologisch sinnvoll und sehr effizient.

\section{Topische Kortikoid-Vitamin- $\mathrm{D}_{3}$-Analoga-Kombinationen}

Aufgrund der pharmakologischen Eigenschaften von Steroiden (Entzündungshemmung) und Vitamin- $\mathrm{D}_{3}$-Analoga (Proliferationshemmung) kann deren Kombination durchaus sinnvoll sein und zu einem schnellen und effektiven Wirkungseintritt führen. Die Fixkombination aus Calcipotriol $(50 \mu \mathrm{g} / \mathrm{g})$ und Betamethasondipropionat $(0,5 \mathrm{mg} / \mathrm{g})$ gilt der Einzelanwendung der Präparate deutlich überlegen $[10,11]$. Zudem reicht eine einmal tägliche Anwendung aus, was die Therapiekosten senkt. Wie oben erwähnt, sollte die gleichzeitige Anwendung von Salizylsäure-haltigen Präparaten während der Anwendung von Calcipotriol-haltigen Präparaten vermieden werden. Um die Steroidnebenwirkungen so gering wie möglich zu halten, kann nach deutlicher Besserung die Kombinationstherapie reduziert oder abgesetzt werden und mit Calcipotriol-haltigen Externa weiterbehandelt werden. Dabei ist ein Rebound im Vergleich zum isolierten Einsatz topischer Steroide selten. Ähnlich wie bei der Therapie mit Vitamin- $\mathrm{D}_{3}$-Analoga bietet sich auch hier die Kombination mit einer Lichttherapie (z. B. mit UVB $311 \mathrm{~nm}$ ) an (siehe Artikel von U. Hofmann).

\section{Dithranol (Cignolin, Anthralin)}

Die Behandlung der Psoriasis mit Dithranol ist seit nunmehr 90 Jahren in der Dermatologie etabliert. Während initial Chrysarobin aus dem Mark von Ararobabäumen isoliert und angewendet wurde, steht uns, erstmals durch Unna and Galewsky seit 1916 eingeführt [12,13], Dithranol (Cignolin, Anthralin) als synthetisches Präparat zur Verfügung. Wenngleich der Wirkmechanismus von Dithranol bis heute nicht bekannt ist und lediglich Einzeleffekte wie Zytostase und Mitosehemmung nachgewiesen wurden [14], ist die klassische bildliche Beschreibung der Wirkungsweise „Die Psoriasis verbrennt im Feuer des Cignolins“ sehr treffend. Denn letztendlich gelingt es unter Einsatz von Cignolin, welches im jeweils therapeutischen Bereich irritierend und somit entzündungsstimulierend wirkt, die pathogenetisch relevante Entzündung der Psoriasis zu verdrängen (Abb. 2). Somit wird Cignolin, obwohl der Wirkmechanismus bis heute nicht klar herausgearbeitet wurde, durchaus dem modernen Verständnis der Rolle eines Immunmodulators gerecht. Von therapeutischem Nutzen ist die Tatsache, dass Cignolin stärker von psoriatischen Plaques als von der umgebenden Haut aufgenommen wird. Deshalb kann z. B. Cignolin in Vaseline oft flächig aufgetragen werden (auf läsionale und nichtläsionale Haut), während Cignolin in Zinkpaste meist zur umschriebenen Anwendung verwendet wird, was je nach Anzahl und Größe der Plaques einen relativ hohen Zeitaufwand beansprucht. Deshalb eignet sich diese Zubereitungsform eher für eine Plaquepsoriasis mit einzelnen großen Herden, weniger aber für eine Psoriasis mit vielen kleinen Einzelherden (Psoriasis guttata). Dem angemischten Cignolinpräparat wird meist 1-2\% Salizylsäure zugesetzt, was eine schnelle Oxidation und somit einen deutlichen Wirkungsverlust
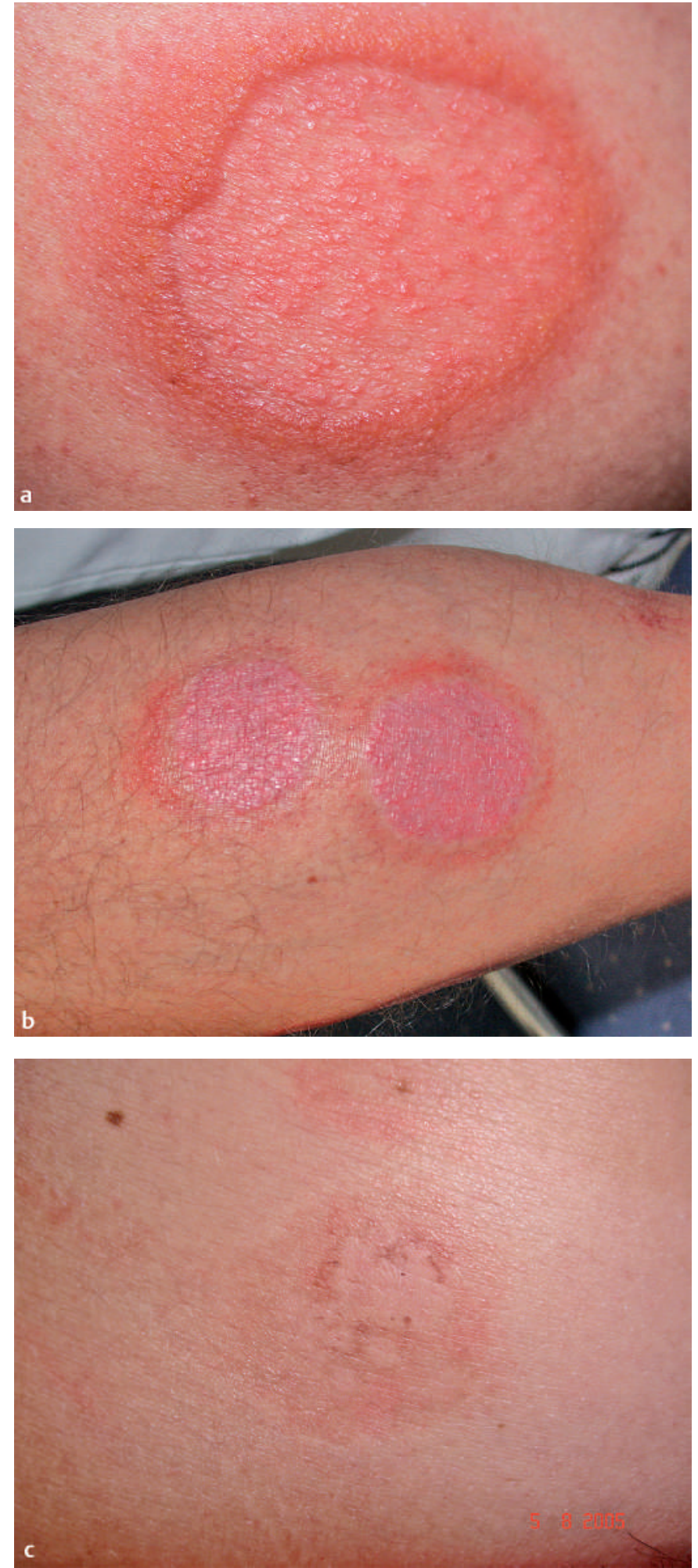

Abb. 2 a Cignolindermatitis: Elevation und Rötung um einen Psoriasisplaque nach 20 -minütiger Anwendung von Micanol ${ }^{\circledR} 1 \%$. b Cignolininduzierte periläsionale leichte Rötung als Nebeneffekt einer richtig dosierten Cignolintherapie („Die Psoriasis verbrennt im Feuer des Cignolins“). c Cignolintherapie: nahezu abgeheilter Psoriasis,plaque“ mit geringer residueller Rötung und leichter Cignolinverfärbung nach Anwendung von Micanol ${ }^{\circledR}$. 
von Cignolin verhindert und zusätzlich die Penetration und klinische Wirksamkeit von Cignolin verstärkt [15]. Bei lokaler Anwendung ist die systemische Toxizität vernachlässigbar [16], weshalb Cignolin auch bei Kindern oder Schwangeren angewendet werden kann (siehe Artikel von Benoit und Goebeler in diesem Heft). Ausnahme ist nur die Brustregion bei stillenden Müttern.

Aufgrund der häufigen Ausbildung einer Cignolindermatitis, die bei sehr empfindlichen Patienten auch schon bei geringen Konzentrationen ausgeprägt verlaufen kann (Abb. 2), muss zu Therapiebeginn die individuelle Irritierbarbeit getestet werden. Obwohl keinerlei gravierenden Nebenwirkungen bekannt sind, verlangt die Anwendung von Cignolin nichtsdestotrotz viel dermatologische Erfahrung und gute Patientenführung. Da eine Cignolindermatitis erst verzögert und v. a. am Rand der Läsion auftritt (Maximum nach 48 Stunden), sollte zu Beginn der Therapie die Cignolinkonzentration niedrig bzw. die Anwendungszeit kurz sein und anfangs nur alle 2 Tage gesteigert werden. Im Prinzip stehen zwei Möglichkeiten der Steigerung zur Verfügung. Zum einen kann die Konzentration des Cignolinpräparates sukzessive erhöht - meist wird mit $0,1 \%$ oder $1 / 16 \%$ (jeweils $1: 1$ Verdünnungsschritte einer $1 \%$ Cignolinzubereitung/1/2\%, $1 / 4 \%, 1 / 8 \%$, $1 / 16 \%$ ) begonnen und Stufe für Stufe bis zu maximal $4-5 \% \mathrm{Ci}$ gnolin gesteigert - oder alternativ die Anwendungszeit (beginnend mit $10 \mathrm{~min}$ ) verlängert werden. Dabei wird nach Ablauf des Zeitintervalls das Präparat abgewaschen und die Läsion anschließend mit pflegenden Externa behandelt (s. o.). Man sollte die Patienten instruieren, dort zuerst abzuwaschen, wo zuerst behandelt wurde. Diese Form der kurzzeitigen lokalen Anwendung wird als sog. Minuten- oder Kurzzeittherapie bezeichnet $[17,18]$ und ist auch für die ambulante Therapie geeignet. Allerdings ist eine gute Compliance der Patienten Voraussetzung. Der Patient sollte darüber informiert sein, dass die leichte randständige Rötung und Braunverfärbung eine notwendige, zur Heilung führende Begleiterscheinung ist (Abb. 2). Das Aushändigen eines Aufklärungsblattes z.B. des Deutschen Psoriasisbundes ist hilfreich. Vor initialen Konzentrationen von 3-4\% Cignolinvaseline für 30-60 min, wie bedauerlicherweise in einem führenden deutschen Dermatologielehrbuch festgehalten, muss ausdrücklich gewarnt werden, da bereits bei Anwendung von einer $1 \% \mathrm{Ci}$ gnolinpräparation für $10 \mathrm{~min}$ in seltenen Fällen eine massive $\mathrm{Ci}$ gnolindermatitis auftreten kann (Abb. 2 b). Der Arzt sollte den Patienten auch im Hinblick auf das braune Oxidationsprodukt des Cignolins auf die Wäscheverfärbung hinweisen. Durch entsprechende Galenik (Verkapselung in semikristallinen Monoglyzeriden) ist in einem Fertigpräparat (Micanol ${ }^{\circledR} 1 \%$ und 3\%) die Oxidation von Cignolin an der Hautoberfläche und der Effekt der Braunverfärbung der Wäsche deutlich reduziert [19]. Eine zusätzliche Effizienzverbesserung kann durch die Kombination mit UVB $311 \mathrm{~nm}$ erzielt werden. Dann sollte Cignolin zur Vermeidung zusätzlicher Reizungen allerdings erst nach Lichttherapie angewendet werden.

Bei pustulösen und insbesondere erythrodermatischen Psoriasisformen ist die Anwendung von Cignolin aufgrund seiner hautreizenden Wirkung nicht empfehlenswert. In den Intertrigines ist die irritierende Wirkung ebenfalls unerwünscht, während Cignolin an der Kopfhaut und bei palmoplantarer Psoriasis erfolgreich eingesetzt werden kann. Die Tatsache, dass auch heute noch die meisten deutschen Hautkliniken im stationären Bereich Cignolin als „First-Line-Therapie“ einsetzen - die Cignolintherapie war bis vor Einführung der Kurzzeittherapie eine Domäne der stationären Behandlung - macht deutlich, welch hoher Stellenwert dieser Therapie zumindest im deutschsprachigen Raum beigemessen wird. Die Cignolintherapie kann mit Fug und Recht bis heute als der „Goldene Standard“ der Psoriasistherapie betrachtet werden. Alle anderen lokalen und systemischen Behandlungsformen müssen sich letztendlich unter dem Gesichtspunkt der Kosteneffizienz und Wirkungseffektivität am Antipsoriatikum Cignolin messen.

\section{Tazaroten}

Seit fast 10 Jahren steht uns in Form von Tazaroten (Zorac Gel ${ }^{\circledR}$ $0,05 \%$ und 0,1\%), einem Rezeptor-selektiven Vitamin-A-Säurederivat, eine weiteres Antipsoriatikum zur Verfügung. Dieses Retinoid reguliert via Rezeptorbindung im Wesentlichen Gene, die für die Proliferation und Differenzierung von Keratinozyten verantwortlich sind [20,21]. Tazaroten wirkt wie die Vitamin$\mathrm{D}_{3}$-Analoga gegen die Akanthose und Hyperkeratose der Psoriasis. Die irritierende Eigenschaft, ähnlich anderer lokaler Retinoide (z.B. Isotretinoin), führte in einer Studie immerhin bei $22 \%$ der Patienten zu Brennen, Juckreiz und Erythem [22]. Daher ist die Akzeptanz von Tazaroten relativ gering und Tazaroten wird im Vergleich zu anderen Antipsoriatika selten rezeptiert. Eine deutliche Wirkungssteigerung kann durch die Kombination mit einer UVB $311 \mathrm{~nm}$-Bestrahlung erzielt werden. Wie bei Vitamin$\mathrm{D}_{3}$-Analoga kann durch die Lichttherapie die entzündliche Komponente, die weder durch Tazaroten noch durch Vitamin$\mathrm{D}_{3}$-Analoga befriedigend behandelt wird, deutlich reduziert bzw. zum Abklingen gebracht werden [23]. Aufgrund der Teratogenität von Retinoiden sollten diese nicht bei Schwangeren und auch nicht während der Stillzeit eingesetzt werden.

Teere

Teerpräparate waren über Jahrzehnte wichtiger Bestandteil der Psoriasisbehandlung. Mit der Einführung lokaler Steroide und weiterer moderner Antipsoriatika ist die Anwendung von Teeren stark rückläufig. Geruch, eine Vielzahl an pharmakologisch wenig definierten Wirksubstanzen, ein eher geringes therapeutisches Potenzial und der Gehalt an potenziell kanzerogenen Substanzen in den Teerdestillaten sprechen gegen eine Monotherapie mit Teeren. Die Kombination von Pix Lithantracis mit einer nachfolgenden UVB-Therapie, bekannt als „Goeckerman-Schema“, führt zu einer deutlichen Effizienzsteigerung und wird vor allem im amerikanischen Sprachraum noch relativ häufig angewendet.

Mahonia aquifolium

Ein Extrakt aus Mahonia aquifolium (Rubisan ${ }^{\circledR}$ Salbe) zeigt eine geringe antipsoriatische Wirkung. Daher ist die Anwendung auf sehr leichte Formen der Psoriasis beschränkt. Ein Argument für die Verwendung von Mahonia aquifolium ist unter Umständen der Wunsch des Patienten nach Alternativpräparaten bzw. Phy- 
Tab. 1 Kombinationstherapie der Psoriasis vulgaris (modifiziert nach Christophers et al. [24]); +++ sehr gut geeignet, ++ gut geeignet, + beschränkt geeignet, - nicht geeignet oder sinnlos

\begin{tabular}{|c|c|c|c|c|c|c|}
\hline & Dithranol & Kortikoide & Salizylsäure & Tazaroten & Vitamin- $D_{3}$-Analoga & UVB 311nm \\
\hline Dithranol & & $-1+$ & ++ & - & - & +++ \\
\hline Kortikoide & - & & ++ & ++ & +++ & +++ \\
\hline Salizylsäure & ++ & ++ & & $-1+$ & - & ++ \\
\hline Tazaroten & - & ++ & $-1+$ & & + & ++ \\
\hline Vitamin-D ${ }_{3}$-Analoga & - & +++ & - & + & & +++ \\
\hline UVB $311 \mathrm{~nm}$ & +++ & ++ & ++ & ++ & +++ & \\
\hline
\end{tabular}

tomedizin. Daher ist die Anwendung von Mahonia aquifolium anderen Phytopräparaten ohne klaren Wirkungsnachweis vorzuziehen.

\section{Kombinationstherapien}

In den letzten Jahren hat sich das Konzept der Kombinationstherapie durch die aufkommende Vielzahl neuer pharmakologischer Substanzen auch für die topische Behandlung der Psoriasis durchgesetzt. Mit Ausnahme der Monotherapie mit Cignolin oder Steroiden sind alle Lokaltherapeutika einschließlich der Lichttherapie nicht ausreichend, um sowohl die Entzündung wie auch die Hyperproliferation zu behandeln. Deshalb macht es Sinn, jeweils jene Lokaltherapeutika zu kombinieren, die entweder antiproliferativ oder antientzündlich wirken (Tab.1). Optimalerweise kommt es durch die Addition von Einzeleffekten zu Synergismen mit der Möglichkeit, durch jeweils geringere Wirkstoffkonzentrationen die Nebenwirkungen deutlich zu reduzieren. Gleichzeitig kann die benötigte Menge an relativ teuren Präparaten (z.B. Vitamin-D ${ }_{3}$-Analoga) begrenzt werden. Sofern man die Lichttherapie auch als Lokaltherapie bewertet, sind insbesondere UVB 311 nm-Bestrahlung und Kortikoide entzündungshemmend und Vitamin- $\mathrm{D}_{3}$-Analoga und Tazaroten antiproliferativ. Dithranol nimmt eine Sonderstellung ein, da die Entzündungshemmung eine indirekte ist und eine Kombinationstherapie eigentlich nur mit UVB $311 \mathrm{~nm}$ sinnvoll ist. Tab. 1 zeigt die geeigneten und ungeeigneten Kombinationen der heute gängigen Antipsoriatika. Die qualitative Bewertung sinnvoller Kombinationen ist sehr schwer, da bis heute klare Therapieleitlinien auf der Basis evidenzbasierter Medizin fehlen und lediglich kleinere Studien, die eigene Erfahrung und das Wissen über den Wirkmechanismus der Therapeutika herangezogen werden können. Die gelegentlich propagierte Sinnhaftigkeit einer Kombination von Cignolin und lokalem Steroid ist deshalb nicht nachzuvollziehen, da ja gerade die Cignolin-provozierte Entzündung die Psoriasis „verbrennt“ und deshalb parallele Entzündungsunterdrückung durch Steroide die Cignolinwirkung eher neutralisiert.

\section{Calcineurininhibitoren}

Nachdem gezeigt wurde, dass Ciclosporin als systemisches Therapeutikum für schwere Psoriasisfälle geeignet ist, sind anfängliche Hoffnungen auf eine lokale Anwendung bald verflogen, weil Ciclosporin nicht in ausreichender Quantität in psoriatische Pla- ques penetriert, um therapeutische Wirkstoffspiegel zu erreichen. Auch die neueren Calcineurininhibitoren (Pimecrolimus ${ }^{\circledR}$, Tacrolimus $^{\circledR}$ ) erreichen nicht die notwendigen therapeutischen Wirkstoffkonzentrationen. Unter quasi okklusiven Effekten, wie z. B. bei Anwendung in der Rima ani, können allerdings leichtere Psoriasisformen behandelt werden. Dabei bietet sich eine intermittierende Anwendung an, um z.B. Steroide einzusparen, die im intertriginösen Bereich mit einem erhöhten Atrophierisiko verbunden sind. Es muss aber darauf hingewiesen werden, dass für den Einsatz von Calcineurininhibitoren bei der Psoriasis keine Zulassung besteht. Die Off-Label-Anwendung muss deshalb mit dem Patienten besprochen und das explizite Einverständnis eingeholt werden.

\section{Alternative Therapien}

Inwiefern alternative bzw. komplementäre Therapieansätze sinnvoll sind oder nicht, darüber lässt sich weidlich streiten. Unbestritten ist, dass die Evidenzen für alternative Therapieverfahren dürftig sind. Unbestritten ist aber auch, dass sich nichtsdestotrotz mehr als die Hälfte der Psoriatiker aus dem innigen Wunsch und dem unseriösen Versprechen einer endgültigen Heilung, und aus teils schlechten Erfahrungen mit der Schulmedizin alternativen bzw. komplementären Therapien zuwendet $[25,26]$. Abwertende Bemerkungen über alternative Therapien werden von Patienten oft weniger geschätzt als detaillierte Aufklärung und Anleitung zur Therapie. Aufklärende Broschüren (z. B. des Dt. Psoriasisbundes) und selbstverfasste Aufklärungszettel helfen dabei, den Patienten zur Mitarbeit zu motivieren. Nur durch die konsequente Anwendung der Lokaltherapeutika und die Einhaltung der Therapievorgaben kann die Psoriasis gut behandelt werden. Gerade für Psoriasispatienten mit einer erheblichen Einschränkung ihrer Lebensqualität hat die sprechende Medizin einen wesentlichen Anteil am Therapieerfolg.

\section{Evidenzbasierte Lokaltherapie der Psoriasis}

Die Wirkungen gängiger Lokaltherapeutika sind allesamt schon aufgrund der Erfahrung des Therapeuten, der großen Zahl an Patienten und an der eindeutigen Ablesbarkeit von klinischen Verbesserungen während der Therapie unstrittig. Der Psoriasispatient hat aber den Anspruch und auch das Recht, von seinem betreuenden Dermatologen zu erfahren, welche (Kombinations-)Therapie für ihn die beste ist. Ein Patient möchte aufgrund 
eines Auftritts in der Öffentlichkeit schnelle Heilung, ein anderer insbesondere ein langes rezidivfreies Intervall und dies auch unter Umständen selbst bei hohem therapeutischen Aufwand. Gemeinsam ist beiden der Wunsch auf eine komplette Heilung, auch wenn dies nur vorübergehend erreicht wird. Jeder erfahrene Dermatologe muss sich deshalb schon wundern, wenn in einer angesehenen Dermatologiezeitschrift unsere Therapiemöglichkeiten klein geredet werden und vermittelt wird, dass eine Abheilung der Psoriasisherde keine realistische Erwartung sein sollte („Clearance is not a realistic expectation of psoriasis treatment“) [27]. Eine komplette Abheilung der Psoriasis ist mit Ausnahme von komplizierten, therapierefraktären Verläufen zweifelsohne das Ziel jeder Behandlung und nicht unrealistisch. Bei der Behandlung mit Cignolin liegt die Abheilquote bei $90 \%$. Aber trotz dieser klaren Evidenzen und den Grund zum ehrlichen therapeutischen Optimismus jedem neuen Psoriasispatienten gegenüber, sind uns weitere Fragen des Patienten über das Für und Wider der einzelnen Therapien aus Unkenntnis wohl eher unangenehm. Der Patient will nicht die mögliche PASI-ScoreVerbesserung wissen, wie sie in vielen Studien als wesentliches Bewertungsprinzip angegeben wird, sondern erfahren, wie hoch seine Chance der kompletten Abheilung ist. Leider sind im Gegensatz zu anderen Volkskrankheiten, zu denen die Psoriasis mit einer Inzidenz von ca. 2 \% gehört, aussagekräftige und insbesondere vergleichende, bewertbare und evidenzbasierte Therapiestudien eine Rarität. Während z. B. in der Diabetologie, Rheumatologie und Kardiologie Studien mit Fallzahlen von oft tausenden Patienten und klaren Evidenzen die Regel sind, gibt es diese für die Therapie der Psoriasis und anderen häufigen chronischen Hautkrankheiten nicht. Bei über 12000 Artikeln zur Psoriasistherapie und über 20000 Artikeln zur evidenzbasierten Medizin (EBM) findet sich gerade einmal eine gemeinsame Schnittmenge von 17 Publikationen. Dabei handelt es sich überwiegend um Übersichtsartikel. Auch Willensbekundungen von akademischer Seite über die Erarbeitung evidenzbasierter Leitlinien zur Psoriasis vulgaris sind zunächst nur Absichtsbekundungen, aber noch keine evidenzbasierte Medizin [26], die dem Patienten und dem praktisch tätigen Dermatologen weiterhelfen. Man muss in Kenntnis der bis dato publizierten Studien schon jetzt vermuten, dass aufgrund der sehr unterschiedlichen Studienpopulationen, unterschiedlicher Anwendung ein und desselben Medikamentes, unterschiedlicher Read-out-Systeme, Nichtbestimmung des rezidivfreien Intervalls und der Lebensqualität etc. weder für den Patienten noch für den Therapeuten diese brennenden Fragen durch Erarbeitung von Leitlinien beantwortet werden. Deshalb wäre es wohl sinnvoller, sich der Konzeption vergleichender Therapiestudien zu widmen, wie es in anderen medizinischen Fachgruppen schon längst geschehen ist. Ziel muss zu guter Letzt die Beantwortung der Fragen des Patienten sein, die da heißen können: Wie lange dauert die Therapie bis zur Abheilung? Wie wahrscheinlich ist die Rate der kompletten Abheilung mit dieser Therapie? Wie lange dauert es, bis erneut Herde auftreten (rezidivfreies Intervall), was sind weitere Voroder Nachteile der Therapie? Erst wenn wir diese Fragen auf dem Boden evidenzbasierter Medizin beantworten können, sind wir auf einem mit anderen medizinischen Fachgruppen vergleichbaren Stand.
Bis dahin gilt es, eigene Erfahrungen durch aufmerksames Vergleichen verschiedener Modalitäten der Lokaltherapie zu sammeln. Dann wird man auch erkennen, dass an einer Studie, die einen Vorteil der Calcipotrioltherapie gegenüber der Cignolintherapie feststellt, möglicherweise etwas nicht stimmen kann [28]. Denn die alltägliche Erfahrung lehrt, dass die Cignolintherapie aufgrund ihrer Verträglichkeit, ihrer Effizienz und insbesondere ihrer relativ langen rezidivfreien Intervalle [29] bis heute den goldenen Standard der topischen Psoriasistherapie darstellt. Der aufmerksame Leser wird bereits schon vorher die Präferenz des Autors für diese alte und doch so moderne Therapie zwischen den Zeilen gelesen haben...

\section{Literatur}

${ }^{1}$ Bernstein JE, Parish LC, Rapaport M et al. Effects of topically applied capsaicin on moderate and severe psoriasis vulgaris. J Am Acad Dermatol 1986; 15: 504-507

${ }^{2}$ Feldmann RJ, Maibach HJ. Absorption of Some organic compounds through the Skin of Man. J Invest Dermatol 1970; 54: 399

${ }^{3}$ Höger PH. Papulosquamaöse Erkrankungen/Psoriasis. Kinderdermatologie 2005; 20: 179

${ }^{4}$ Endzweig-Gribetz CH, Brady C, Lynde C et al. Drug interactions in psoriasis: the pros and cons of combining topical therapies. J Cutan Med Surg 2002; 6 (3 Suppl): $12-16$

${ }^{5}$ Morimoto S, Kumahara Y. Patient with Psoriasis Cured by 1-Alpha-hydroxy-vitamin D3. Med J Osaka Uni 1985; 35: 51

${ }^{6}$ Mee JB, Cork MJ. Vit D receptor polymorphism and calcipotriol response in patients with psoriasis. J Invest Dermatol 1998; 110: 301 302

${ }^{7}$ Van de Kerkhof PCM, Vissers WHPM. The topical treatment of psoriasis. Skin Pharmacol Appl Skin Physiol 2003; 16: 69-89

${ }^{8}$ Lagner A, Ashton P, van de Kerkhoff OC, Verjans H. A long-term multicenter assessment of the safety of calcipotriol ointment in the treatment of chronic plaque psoriasis. Br J Dermatol 1996; 135: 385 - 389

${ }^{9}$ Van de Kerkhoff PCM, Berth-Jones J, Griffiths CEM et al. Long-term efficacy and safety of tacalcitol ointment in patients with chronic plaque psoriasis. Br J Dermatol 2002; 146: 414-422

10 Guenther L, Cambazard F, van de Kerkhoff PCM. Efficacy and safety of a new combination of calcipotriol and betamethasone dipropionate (once or twice daily) compared to calcipotriol (twice daily) in the treatment of psoriasis vulgaris: a randomized, double-blind vehiclecontrolled clinical trial. Br J Dermatol 2002; 147: 316-323

${ }^{11}$ Papp KA, Guenther L, Boyden B et al. Early onset of action and efficacy of a combination of calcipotriol and betamethasone dipropionate in the treatment of psoriasis. J Am Acad Dermatol 2003; 48: 48 -54

12 Galewski E. Über Cignolin, ein Ersatzpräparat des Chrysarobins. Dermatol Wochenschr 1916; 62: 113-115

${ }^{13}$ Unna PG. Cignolin als Heilmittel der Psoriasis. Dermatol Wochenschr 1916; 62 : $116-137,150-163,175-183$

${ }^{14}$ Klem EB. Effects of antipsoriatic drugs and metabolic inhibitors on the growth of epidermal cells in culture. J Invest Dermatol 1978; 70: 27 32

${ }^{15}$ Ippen H. Basic questions concerning toxicology and pharmacology of anthralin. 1. CIRD Symposium: Anthralin, Sophia Antipolis Frankreich: 1980

${ }^{16}$ Lukacs S, Braun-Falco O. Über das Verhalten von Dithranol in Pasten und Lösungen und seine Beeinflussung durch Salizylsäure. Hautarzt 1973; 27: 304-309

${ }^{17}$ Kunze J, Runne U. Minutes Therapy with Anthralin for Psoriatic OutPatients. Psoriasis. Proceedings of the 3rd International Symposium, Stanford University. New York Grune and Stratton, 1982

${ }^{18}$ Schauder S, Mahrle G. Kombinierte 1-Stunden-Therapie der Psoriasis mit Anthralin und UV-Licht. Hautarzt 1982; 33: 206-209

${ }^{19}$ Volden G, Björnberg A, Tegner E, Pedersen NB et al. Short-contact treatment of psoriasis at home with Micanol ${ }^{\circledR}$. Acta Derm Venerol 1992; suppl 172: $20-22$

${ }^{20}$ Chandraratna RA. Tazarotene: first of a new generation of receptor-selective retinoids. Brit J Dermatol 1996; 135 (Suppl 49): 18-25 
${ }^{21}$ Weinstein GD. Safety, efficacy and duration of therapeutic effect of tazarotene used in the treatment of plaque psoriasis. Br J Dermatol 1996; 135 (Suppl 49): $32-36$

${ }^{22}$ Krueger GG, Drake LA, Elias PM et al. The safety and efficacy of tazarotene gel, a topical acetylenic retinoid, in the treatment of psoriasis. Arch Dermatol 1998; 134: 57-60

${ }^{23}$ Koo JY. Tazarotene in combination with phototherapy. J Am Acad Dermatol 1998; 39: S144-S148

${ }^{24}$ Christophers E, Mrowietz U, Sterry W. Psoriasis auf einen Blick. Berlin: Blackwell, 2003
${ }^{25}$ Ben-Arye E, Ziv M, Frenkel M et al. Complementary medicine and psoriasis: linking the patient's outlook with evidence-based medicine. Dermatology 2003; 207: 302 - 307

${ }^{26}$ Capella GL, Finzi AF. Complementary therapy in psoriasis. Dermatol Ther 2003; 16: 164-174

${ }^{27}$ A-Suwaidan SN, Feldmann SR. Clearance is not a realistic expectation of psoriasis treatment. J Am Acad Dermatol 2000; 42: 796-802

${ }^{28}$ Dutz JP, Lui HA. A comparative study of calcipotriol and anthralin for chronic plaque psoriasis in a day care treatment center. Int J Dermatol 1998; 37: $51-55$

${ }^{29}$ Koo J, Lebwohl M. Duration of remission of psoriasis therapies. J Am Acad Dermatol 1999; 41: 51 - 59

\section{Buchbesprechung}

\section{Neurofibromatose Typ 1. Die Rolle der bildgebenden Diagnostik}

B. Nenning, P. Gutjahr (Hsg.)

Köln: Deutscher Ärzte-Verlag, 2006. 132 S., 105 Abb., Kart.

$39,95 €$, ISBN 3-7691-1204-0

Die vor über 120 Jahren erstmals von Recklinghausen als eigenes Krankheitsbild beschriebene Neurofibromatose ist als Typ I (NF 1) mit einer Inzidenz von 1 auf 2500 bis 4000 Lebendgeborene in Deutschland das häufigste erbliche Tumordispositionssyndrom. Es ist gekennzeichnet durch die Entwicklung multipler gutartiger Nervenscheidentumoren, der Neurofibrome, Pigmentveränderungen der Haut, den sogenannten Café-au-lait-Flecken, Iris- und Sehnervenveränderungen, charakteristische Skelettveränderungen sowie Lernstörungen und Intelligenzabweichungen. Die Herausgeber der vorliegenden Monographie betreuen in der Onkologischen Ambulanz der Universitätskinderklinik Mainz über 120 Patienten mit einer NF 1, so dass sie über ein reichhaltiges Erfahrungsgut verfügen, das diesem Buch zugrunde liegt. In den ersten fünf Kapiteln wird ein prägnanter Überblick über die Neuropathologie, Molekulargenetik und die Klinik gegeben. Dem folgt als erstes bildgebendes Verfahren das von R. Schumacher verfasste Kapitel über die konventionelle Röntgendiagnostik. Mit einducksvollen Bildbeispielen werden die hierbei im Bereich des Schädels, der Wirbelsäule, im Becken- und Thoraxbereich sowie an den Extremitäten auftretenden Veränderungen beschrieben. Während es sich hierbei überwiegend um indirekte Zeichen handelt, die durch den Druck von Neurofibromen oder anderen bei der NF 1 vorkommenden Tumoren auf benachbarte Skelettabschnitte bewirkt werden, ist deren direkter Nachweis durch die moderne Schnittbilddiagnostik möglich.

Das von R. Brzezinska verfasste Kapitel über die Ultraschalldiagnostik bei NF 1 zeigt die inzwischen erreichte Qualität mit Abbildungen von erstaunlicher Aussagekraft. Ist diese im Bereich der Kutis und Subkutis beim Nachweis von Neurofibromen nicht unerwartet, überrascht die Leistungsfähigkeit im Bereich des ZNS. Vor allem im Orbitatrichter lassen sich die Papille sowie der retrobulbäre Verlauf des Nervus opticus schon als normale Struktur gut nachweisen. Sehr gut gelingt daher die Darstellung von Optiukusgliomen und ihrer Folgezustände wie die Ausbildung einer Stauungspapille. Auch der Nachweis abdominaler und retroperitonealer Neurofibrome ist dem sonographisch versierten Untersucher gut möglich.

K. Brühl demonstriert in einem neuroradiologischen Kapitel die große Aussagekraft der Kernspintomographie bei der NF 1. Dabei sind die bei Kindern vorkommenden Signalhyperintensitäten in den Stammganglien schwieriger nachzuweisen. Gliome lassen sich in der Regel im Gegensatz zur Sonographie im Verlauf der gesamten Sehbahn darstellen. Die zerebralen Gefäße weisen neben Stenosen auch Aneurysmen und AV-Malformationen auf. Letztere lassen sich häufig mit der MR-Angiographie darstellen, die konventionelle Angiographie ist daher aus diagnostischer Indikation nur noch selten erforderlich. Ein über 20 Seiten umfassender Bildteil veranschaulicht eindrucksvoll die Leistungsfähigkeit der Kernspintomographie und gibt dabei wertvolle Hinweise auf die Untersuchungstechnik. Auch im Spinalbereich erscheint die MR der CT eindeutig überlegen.

Kreitner und Gutjahr zeichnen für das Kapitel der NF 1 mit Manifestationen außerhalb des ZNS verantworlich. Hier ergänzen sich MR und CT, indem sie die topographischen Beziehungen zu benachbarten Strukturen aufzeigen. Obwohl Nervenscheidentumoren im CT eher hypodens erscheinen und nach KM-Verstärkung an Dichte deutlich zunehmen, ist wie auch mit der MR eine sichere Abgrenzung gegen andersartige Läsionen häufig nicht möglich. Auch die sarkomatöse Entartung von Neurofibromen lässt sich nicht immer erkennen.

Die letzten Kapitel des vorliegenden Buchs befassen sich mit urologischen und neurochirurgischen Aspekten der NF 1. So können größere Neurofibrome Ursachen von Obstruktionen des oberen Harntrakts oder auch von Miktionsbeschwerden sein, wenn sie im kleinen Becken auftreten. Eine Hypertonie ist sowohl durch eine bei NF 1 häufigere Nierenarterienstenose als auch durch Assoziation der NF mit einem Phäochromozytomen möglich. Ausführlicher wird auf operative Behandlungsmöglichkeiten von urologischer Seite eingegangen. Die neurochirurgischen Therapiemöglichkeiten werden am Beispiel der Gliome der Sehbahn dargestellt.

Insgesamt stellt die vorliegende Monographie eine kompakte Übersicht für die mit der Neurofibromatose befassten Ärzte verschiedener Fachdisziplinen dar. Dem Dermatologen werden richtungsweisende Hinweise auf das bildgebende Staging gegeben. Der Radiologe bekommt in einer prägnanten Übersicht die heute einzusetzenden Verfahren mit ihrer speziellen Untersuchungstechnik sowie typische Befunde an sehr guten Bildbeispielen vermittelt. Auch Orthopäden, Urologen und Neurochirurgen dürften zu den Nutzern gehören, die von dem vorgelegten Leitfaden profitieren.

M. Georgi, Heidelberg 